\title{
COMPACT TOEPLITZ OPERATORS WITH CONTINUOUS SYMBOLS ON WEIGHTED BERGMAN SPACES
}

\author{
TAKAHIKO NAKAZI* and RIKIO YONEDA \\ Department of Mathematics, Faculty of Science, Hokkaido University, Sapporo 060-0810, Japan
}

(Received 26 March, 1998)

\begin{abstract}
Let $L_{a}^{2}(D, d \sigma d \theta / 2 \pi)$ be a complete weighted Bergman space on the open unit disc $D$, where $d \sigma$ is a positive finite Borel measure on $[0,1)$. We show the following: when $\phi$ is a continuous function on the closed unit disc $\bar{D}, T_{\phi}$ is compact if and only if $\phi=0$ on $\partial D$.
\end{abstract}

1991 Mathematics Subject Classification. 47B35, 47B07

Let $D$ be the open unit disc and $d \sigma$ a positive finite Borel measure on $[0,1)$. Let $L_{a}^{2}=L_{a}^{2}(D, d \sigma d \theta / 2 \pi)$ be a weighted Bergman space on $D$; that is, $L_{a}^{2}$ consists of analytic functions $f$ in $D$ with

$$
\|f\|_{2}^{2}=\int_{D}\left|f\left(r e^{i \theta}\right)\right|^{2} d \sigma d \theta / 2 \pi<\infty
$$

When $L_{a}^{2}$ is closed, $P$ denotes the orthogonal projection from $L^{2}=L^{2}(D, d \sigma d \theta / 2 \pi)$ onto $L_{a}^{2}$. For $\phi$ in $L^{\infty}=L^{\infty}(D, d \sigma d \theta / 2 \pi)$, we consider the Toeplitz operator $T_{\phi}: L_{a}^{2} \rightarrow L_{a}^{2}$ defined by $T_{\phi} f=P(\phi f), f \in L_{a}^{2}$. We prove the following theorem in this paper. For the Bergman space (that is, $d \sigma=2 r d r$ ), the Theorem is well known; see [5, p. 107] and [1]. When $d \sigma=\left(1-r^{2}\right)^{\alpha} d r(-1<\alpha<\infty)$, the Theorem is also true; see [3] and [4]. However, that argument does not work for the general situation. We need a new idea in order to prove the Theorem. Let $H=H(D)$ denote the set of all analytic functions on $D$.

Theorem. Suppose that $L_{a}^{2}=L_{a}^{2}(D, d \sigma d \theta / 2 \pi)$ is complete. When $\phi$ is a continuous function on the closed unit disc $\bar{D}, T_{\phi}$ is compact if and only if $\phi=0$ on $\partial D$.

In order to prove the Theorem, we need three lemmas.

LeMma 1. $L_{a}^{2}$ is complete if and only if $\sigma([\varepsilon, 1))>0$ for some $\varepsilon$ with $0 \leq \varepsilon<1$.

Proof. For $a \in D$, put

$$
s(\mu, a)=\inf \left\{\int_{D}|f|^{2} d \mu ; f \in H \text { and } f(a)=1\right\}
$$

*This research was partially supported by Grant-in-Aid for Scientific Research, Ministry of Education. 
where $H$ is the set of all analytic functions on $D$ and $d \mu=d \sigma d \theta / 2 \pi$. Statement (1) of Corollary 1 in [2] is valid for $s(\mu, a)$ instead of $S(\mu, a)$. When $(\operatorname{supp} \mu) \cap D$ is a uniqueness set for $H$, by Statement (1) of Theorem 8 in [2], $L_{a}^{2}$ is complete if and only if, for all compact sets $K$ in $D, \int_{K} \log s(\mu, a) r d r d \theta / \pi>-\infty$. If $\sigma$ is not a zero measure, then $(\operatorname{supp} \mu) \cap D$ is a uniqueness set for $H$. These statements suffice to prove the Lemma.

Lemma 2. If $\sigma([\varepsilon, 1))>0$ for every $\varepsilon$ with $0 \leq \varepsilon<1$, then

$$
\lim _{n \rightarrow \infty} \frac{\int_{0}^{\varepsilon} r^{n} d \sigma}{\int_{\varepsilon}^{1} r^{n} d \sigma}=0 \quad(0 \leq \varepsilon<1) .
$$

Proof. When $\delta$ is a positive constant with $\varepsilon+\delta<1$, the following inequality holds.

$$
\begin{aligned}
\frac{\int_{0}^{\varepsilon} r^{n} d \sigma}{\int_{\varepsilon}^{1} r^{n} d \sigma} & \leq \frac{\sigma([0, \varepsilon])}{\int_{\varepsilon}^{1}\left(\frac{r}{\varepsilon}\right)^{n} d \sigma} \leq \frac{\sigma([0, \varepsilon])}{\int_{\varepsilon+\delta}^{1}\left(\frac{r}{\varepsilon}\right)^{n} d \sigma} \\
& \leq \frac{\sigma([0, \varepsilon])}{\left(\frac{\varepsilon+\delta}{\varepsilon}\right)^{n} \sigma([\varepsilon+\delta, 1])} \quad(0<\varepsilon<1) .
\end{aligned}
$$

Since they are positive and $\lim _{n \rightarrow \infty}\{(\varepsilon+\delta) / \varepsilon\}^{n}=\infty$, we have

$$
\lim _{n \rightarrow \infty}\left(\int_{0}^{\varepsilon} r^{n} d \sigma / \int_{\varepsilon}^{1} r^{n} d \sigma\right)=0 .
$$

Lemma 3. If for every $\varepsilon$ with $0 \leq \varepsilon<1$, we have

$$
\int_{\varepsilon}^{1} r^{n} d \sigma>0 \text { and } \lim _{n \rightarrow \infty} \frac{\int_{0}^{\varepsilon} r^{n} d \sigma}{\int_{\varepsilon}^{1} r^{n} d \sigma}=0,
$$

then for any non-negative $\ell$

$$
\lim _{n \rightarrow \infty} \frac{\int_{0}^{1} r^{n+\ell} d \sigma}{\int_{0}^{1} r^{n} d \sigma}=1 .
$$


Proof. For every $\varepsilon$ with $0 \leq \varepsilon<1$, the following inequality holds.

$$
\begin{aligned}
1 \geq \frac{\int_{0}^{1} r^{n+\ell} d \sigma}{\int_{0}^{1} r^{n} d \sigma} & =\frac{\int_{0}^{\varepsilon} r^{n+\ell} d \sigma+\int_{\varepsilon}^{1} r^{n+\ell} d \sigma}{\int_{0}^{\varepsilon} r^{n} d \sigma+\int_{\varepsilon}^{1} r^{n} d \sigma} \\
& \geq \frac{\varepsilon^{\ell} \int_{\varepsilon}^{1} r^{n} d \sigma}{\int_{\varepsilon}^{1} r^{n} d \sigma+\int_{0}^{\varepsilon} r^{n} d \sigma} \\
& =\varepsilon^{\ell}\left(1+\frac{\int_{0}^{\varepsilon} r^{n} d \sigma}{\int_{\varepsilon}^{1} r^{n} d \sigma}\right)^{-1}
\end{aligned}
$$

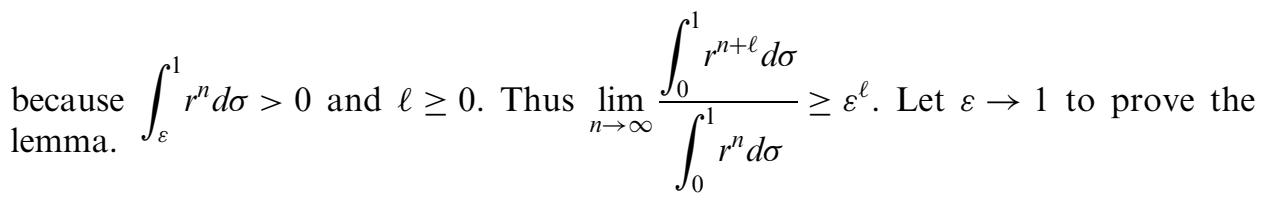
Proof. Suppose that $\phi\left(r e^{i \theta}\right)=\sum_{j=-\infty}^{\infty} \phi_{j}(r) e^{i j \theta}$ is continuous on $\bar{D}$, where

$$
\phi_{j}(r)=\int_{0}^{2 \pi} \phi\left(r e^{i \theta}\right) e^{-i j \theta} d \theta / 2 \pi
$$

for $j=0, \pm 1, \pm 2, \cdots$. Then $\phi_{j}(r)$ is continuous on $[0,1]$ for any $j$. Put

$$
\begin{aligned}
e_{n}\left(r e^{i \theta}\right) & =a_{n} r^{n} e^{i n \theta} \\
& =r^{n} e^{i n \theta} / \sqrt{\int_{0}^{1} r^{2 n} d \sigma}
\end{aligned}
$$

for $n \geq 0$, then $\left\{e_{n}\right\}$ is an orthonormal basis in $L_{a}^{2}$. For each $j$, put

$$
\Phi_{j}\left(r e^{i \theta}\right)=r^{|j|} e^{-i j \theta} \phi\left(r e^{i \theta}\right)
$$

Then $T_{\Phi_{j}}=T_{r|j| e^{-i j \theta}} T_{\phi}$ for $j \geq 0$ and $T_{\Phi_{j}}=T_{\phi} T_{r|j|} e^{-i j \theta}$ for $j<0$. If $T_{\phi}$ is compact, then $T_{\Phi_{j}}$ is also compact for any $j$. For each $j$, if $n \geq 0$, then

$$
\left|\left\langle T_{\Phi_{j}} e_{n}, e_{n}\right\rangle\right| \leq\left\|T_{\Phi_{j}} e_{n}\right\|_{2}\left\|e_{n}\right\|_{2}=\left\|T_{\Phi_{j}} e_{n}\right\|_{2} .
$$

Since $T_{\Phi_{j}}$ is compact for each $j$ and $e_{n} \rightarrow 0(n \rightarrow \infty)$ weakly, $\left\|T_{\Phi_{j}} e_{n}\right\|_{2} \rightarrow 0(n \rightarrow \infty)$ and so $\left\langle T_{\Phi_{j}} e_{n}, e_{n}\right\rangle \rightarrow 0(n \rightarrow \infty)$. For each $j$, 


$$
\begin{aligned}
\left\langle T_{\Phi_{j}} e_{n}, e_{n}\right\rangle & =\int_{0}^{2 \pi} \int_{0}^{1} \phi\left(r e^{i \theta}\right) r^{|j|} e^{-i j \theta} a_{n}^{2} r^{2 n} d \sigma d \theta / 2 \pi \\
& =a_{n}^{2} \int_{0}^{1} \phi_{j}(r) r^{|j|+2 n} d \sigma
\end{aligned}
$$

and then $\lim _{n \rightarrow \infty} a_{n}^{2} \int_{0}^{1} \phi_{j}(r) r^{|j|+2 n} d \sigma=0$. By Lemma $1, \sigma([\varepsilon, 1))>0$ for some $\varepsilon$ with $0 \leq \varepsilon<1$ and hence $\sigma([\varepsilon, 1))>0$ for every $\varepsilon<1$. Hence, by Lemma 2, we have

$$
\lim _{n \rightarrow \infty} \frac{\int_{0}^{\varepsilon} r^{2 n} d \sigma}{\int_{\varepsilon}^{1} r^{2 n} d \sigma}=0 \text { for }(0 \leq \varepsilon<1) .
$$

Then, by Lemma 3, for any integer $j$ we have

$$
\lim _{n \rightarrow \infty} a_{n}^{2} \int_{0}^{1} r^{|j|+2 n} d \sigma=1 .
$$

Since $\phi_{j}(r)$ is continuous on $[0,1]$, we can approximate $\phi_{j}(r)$ uniformly by polynomials $\sum_{t=0}^{k} c_{t} r^{t}$. Since $\lim _{n \rightarrow \infty} a_{n}^{2} \int_{0}^{1} r^{|j|+2 n} d \sigma=1$ for any $j$, we obtain

$$
\lim _{n \rightarrow \infty} a_{n}^{2} \int_{0}^{1}\left(\sum_{t=0}^{k} c_{t} r^{t}\right) r^{|j|+2 n} d \sigma=\sum_{t=0}^{k} c_{t}
$$

and so

$$
\lim _{n \rightarrow \infty} a_{n}^{2} \int_{0}^{1} \phi_{j}(r) r^{|j|+2 n} d \sigma=\phi_{j}(1) .
$$

Thus $\phi_{j}(1)=0$ for any $j$ because $\lim _{n \rightarrow \infty} a_{n}^{2} \int_{0}^{1} \phi_{j}(r) r^{|j|+2 n} d \sigma=0$, and hence $\phi=0$ on $\partial D$.

Conversely suppose that $\phi=0$ on $\partial D$. Then we may assume that the support set of $\phi$ is compact in $D$. In order to show the compactness of $T_{\phi}$, it is sufficient to show that if $h_{n} \rightarrow 0$ weakly $(n \rightarrow \infty)$ in $L_{a}^{2}$ then $h_{n} \rightarrow 0$ uniformly on supp $\phi$. By hypothesis on $\sigma$, any point $z \in D$ has a bounded point evaluation for $L_{a}^{2}$ because Statement (1) of Corollary 1 in [2] is valid for $s(\mu, a)$ instead of $S(\mu, a)$ and $r(\mu, a) s(\mu, a)=1(a \in D)$. Hence $h_{n}(z) \rightarrow 0$. By the boundedness of analytic functions on supp $\phi$ and the uniform boundedness principle, $h_{n} \rightarrow 0$ uniformly on supp $\phi$.

\section{REFERENCES}

1. S. Axler and D. C. Zheng, Compact operators via the Berezin transform, Indiana Univ. Math. J. 47 (1998), 387-400. 
2. T. Nakazi and M. Yamada, Riesz's functions in weighted Hardy and Bergman spaces, Canad. J. Math. 48 (1996), 930-945.

3. K. Stroethoff, Compact Toeplitz operators on Bergman spaces, Math. Proc. Cambridge Phil. Soc. 124 (1998), 151-160.

4. K. Stroethoff, Compact Toeplitz operators on weighted harmonic Bergman spaces, $J$. Australian Math. Soc. Ser. A 64 (1998), 136-148.

5. K. Zhu, Operator theory in function spaces (Dekker, New York, 1990). 